\title{
Dysrhythmia: a specific congenital rhythm perception deficit
}

\author{
Jacques Launay ${ }^{1}$, Manon Grube ${ }^{2}$ and Lauren Stewart ${ }^{3}$ * \\ ${ }^{1}$ Department of Experimental Psychology, University of Oxford, Oxford, UK \\ ${ }^{2}$ Auditory Group, Institute of Neuroscience, The Medical School, Newcastle University, Newcastle-upon-Tyne, UK \\ ${ }^{3}$ Goldsmiths College, University of London, London, UK
}

\section{Edited by:}

Chris Muller, Ghent University, Belgium

\section{Reviewed by:}

Patricia E. G. Bestelmeyer, Bangor University, UK

Robert J. Ellis, Beth Israel Deaconess Medical Center, USA

\section{*Correspondence:}

Lauren Stewart, Goldsmiths College, University of London, New Cross, London SE14 6NW, UK e-mail: I.stewart@gold.ac.uk
Why do some people have problems "feeling the beat"? Here we investigate participants with congenital impairments in musical rhythm perception and production. A web-based version of the Montreal Battery of Evaluation of Amusia was used to screen for difficulties with rhythmic processing in a large sample and we identified three "dysrhythmic" individuals who scored below cut-off for the rhythm subtest, but not the pitch-based subtests. Follow-up testing in the laboratory was conducted to characterize the nature of both rhythm perception and production deficits in these dysrhythmic individuals. We found that they differed from control participants when required to synchronize their tapping to an external stimulus with a metrical pulse, but not when required to tap spontaneously (with no external stimulus) or to tap in time to an isochronous stimulus. Dysrhythmics exhibited a general tendency to tap at half the expected tempo when asked to synchronize to the beat of strongly metrical rhythms. These results suggest that the individuals studied here did not have motor production problems, but suffer from a selective rhythm perception deficit that influences the ability to entrain to metrical rhythms.

Keywords: rhythm, meter, beat, motor timing, amusia

\section{INTRODUCTION}

Rhythm perception and entrainment abilities develop early in human life (Hannon and Trehub, 2005; Phillips-Silver and Trainor, 2005; Zentner and Eerola, 2010) and have been suggested to be relevant to a range of functions, including mother-infant communication (Beebe and Lachmann, 1988; Bernieri et al., 1988; Dissanayake, 2000), speech and language development (Smith et al., 1989; Jusczyk et al., 1992, 1999; Huss et al., 2011; Grube et al., 2012), and social bonding (Knoblich et al., 2011). The entrainment of our body movements to a regular beat enables us to synchronize with the movements of others - a phenomenon which is argued to foster empathy (Hove and Risen, 2009; Wiltermuth and Heath, 2009; Launay et al., 2013) and can have physiological effects such as co-ordinating bodily rhythms with those of others (Konvalinka et al., 2012; Vickhoff et al., 2013) or facilitating organized motor behavior in neurological patients with movement disorders (Thaut and Abiru, 2010).

The ability to perceive and entrain to a regular beat is considered to be a common and highly automatized human ability that can be measured using perceptual and motor tasks, both behaviourally and with neuroimaging techniques (Praamstra et al., 2003; Repp, 2005; Oullier etal., 2008). There has been recent interest in the identification and investigation of individuals who appear to have difficulty with these abilities, but knowledge remains predominantly anecdotal. The few existing scientific attempts to characterize congenital rhythm impairments are typically based on motor measures (e.g., Iversen and Patel, 2008; PhillipsSilver et al., 2011; Farrugia et al., 2012; Sowinski and Dalla Bella, 2013) and more research is required to investigate these kinds of difficulties.
The most simple form of entrainment to a sensory (typically auditory) rhythmic stimulus involves perceiving and synchronizing movements with an isochronous beat with one level of periodicity, such as that produced by a metronome (Wing and Kristofferson, 1973; ten Hoopen et al., 1994; Friberg and Sundberg, 1995; Ehrle and Samson, 2005). Individuals are further able to extract the underlying beat from more complex rhythmic structures with a metrical hierarchy comprised of two or more levels of periodicities that are multiples of each other (London, 2004), and typically tend to tap along at a level corresponding to a preferred periodicity of 400 to 800 milliseconds, peaking around $600 \mathrm{~ms}$ (Fraisse, 1963).

The induction of a perceived beat, or meter, in the listener by the use of temporal cues alone (i.e., in the absence of changes in pitch or sound intensity) has been demonstrated to rely upon certain phenomenal principles. Phenomenal accents are found to occur on tones that are followed or preceded by a relatively long "pause," i.e., time between consecutive event onsets or interonset-intervals. The feeling of a meter can be induced by rather simple rhythmic sequences of identical tones, if those phenomenal accents occur regularly at a multiple of the underlying beat unit, for instance on every fourth beat (Povel and Essens, 1985). In contrast to such strongly metrical sequences, weakly metrical ones in which accented tones do not occur at regular intervals will not induce the "feeling of a beat" in a naïve listener. A number of psychophysical studies have demonstrated an improvement in objective measures of perceptual accuracy and the subjectively perceived "feeling of the beat" or "catchiness" for metrically strong compared to weak sequences (Povel and Essens, 1985; Hirsh et al., 1990; Monahan and Hirsh, 1990; Handel, 1998; Hebert and Cuddy, 2002; Grube 
and Griffiths, 2009). Similarly, performance in synchronization tasks is more accurate for strongly metrical sequences compared to those that are weakly metrical (Patel et al., 2005). Whilst the ability to extract a beat from a complex auditory stimulus has been demonstrated repeatedly in groups of typically developing subjects, there has been little systematic investigation into congenital deficits in beat extraction and entrainment for such stimuli.

Individuals with a developmental disorder termed "congenital amusia," or "tone deafness," are characterized by deficits in the perception of pitch-related features in musical melodies, which may or may not be accompanied by deficits in the perception of rhythm-related features. The diagnostic tool, the Montreal Battery of Evaluation of Amusia (MBEA; Ayotte et al., 2002; Peretz et al., 2003) was developed as a formal test of congenital amusia. Using short musical melodies, the MBEA looks at six separate aspects of music perception including both pitch and rhythm perception tests. Peretz et al. (2003) noted that problems with pitch perception were universal in their cohort of amusic subjects, while rhythm perception was only affected in a subsample of the group. Subsequent studies have further supported the notion that congenital amusia is typically a selective pitch impairment in which rhythm deficits, where present, reflect concomitant effects of the primary pitch deficit (e.g., Peretz and Hyde, 2003; Hyde and Peretz, 2004). In a study specifically testing this hypothesis, pitch-based amusic subjects were shown to perform poorly also in rhythm discrimination tasks, but only for stimuli containing pitch variations (Foxton et al., 2006). Similarly, individuals with amusia have been demonstrated to have problems finding the beat in a musical context: when asked to move in time with musical sounds they would tap at half the expected meter (Dalla Bella and Peretz, 2003). However, the characterization of congenital amusia as a disorder of pitch, rather than rhythm, may partly reflect a screening bias, since individuals sought for such studies have typically been recruited on the basis that they self-report as "tone-deaf" and have trouble singing in tune.

Efforts to seek out people whose predominant difficulty lies in keeping in time, be it in conjunction with pitch deficits or in isolation, have been few and far between. A recent study reported on individuals who had problems with synchronization but not with rhythm perception (Sowinski and Dalla Bella, 2013). Phillips-Silver etal. (2011) investigated one particular "beat-deaf" case ("Mathieu"), who complained about an inability to find the beat in music and exhibited specific difficulties with the meter-identification task of the MBEA and in synchronizing his dance movements with a musical beat (meringue) but not with a metronome (Phillips-Silver et al., 2011). However, this study did not include a systematic investigation of metrical-beat extraction and motor synchronization with controlled auditory stimuli rather than music, leaving questions about the precise locus of the deficit unanswered.

In the present study we sought out individuals who exhibited specific impairments in rhythm perception according to the MBEA (administered on-line) and also self-reported difficulties with rhythm in everyday life. These individuals, whom we subsequently refer to as "dysrhythmic" were then tested to assess their ability to produce an isochronous tapping pulse (i) spontaneously, (ii) in time to isochronous stimulus sequences, and (iii) to sequences with strongly and weakly metrical-beats. In order to investigate the impact of pitch on these synchronization abilities, all three types of sequence were presented with and without pitch variation.

\section{MATERIALS AND METHODS PARTICIPANTS}

The MBEA was used to identify individuals with impairments that were specific to the rhythm subtest of the MBEA and associated with normal performance on the pitch-related subtests (scale, contour, interval). An online version of the scale and rhythm subtests was taken by 89,000 participants, and individuals scoring below the published cut-off scores for the rhythm subtest and above the cut-off score for the scale test (Peretz et al., 2003) were identified. Individuals scoring in this way were asked to retake these two online tests. Provided they continued to score below and above cut-off for the rhythm and scale subtests respectively and they self-reported as having difficulties with rhythm in everyday life, they were invited to the laboratory for testing on four of the MBEA subtests (scale, contour, interval, and rhythm) to verify that their profile of scores was robust. Three individuals were identified: SS, female, aged 26; SWI, male, aged 43; and VPO, female, aged 29 and included in the main study.

Thirty eight control participants (18M, 20F; age range 19-61, mean $=38.92$ ) were also identified from the online database for further inclusion in the present study. These individuals had scored above the published cut-off scores, both for the online MBEA subtests (scale and rhythm) as well as during laboratory-based testing (scale, contour, interval, rhythm subtests). In the tapping tasks, data for two dysrhythmic participants were not recorded for one trial due to a technical error; similarly, data were not recorded for eight trials in one control participant so that particular participant's data are not included in any synchronization task analysis. All participants were reimbursed for travel expenses, and additionally received $\mathfrak{£} 7.50$ for their time (approximately one hour).

\section{EQUIPMENT}

All tapping tasks were performed using a DELL XPS M1530 computer running MAX/MSP 4.5 software. Stimuli were played to participants using an external Alesis IO2 soundcard and Sennheiser HD 265-1 headphones. Participants tapped on the computer keyboard using their preferred hand.

\section{STIMULI}

The strongly and weakly metrical rhythms used in the tapping tasks were of the same type as those developed by Grube and Griffiths (2009), following the phenomenally based rules of metrical-beat induction described by Povel and Essens (1985). Strongly metrical rhythms had accented tones on all four downbeat locations for a meter of 4 (i.e., units 1, 5, 9, 13 of 16) while weakly metrical rhythms had accented tones in only two of those hypothetical downbeat locations (first and last) and two silent beat locations in-between (second and third). Both sets of rhythms have a larger 


Isochronous
Weakly
metrical
metrical
[960]
stguRE 1 I Schematic representation of tapping stimuli: isochronous,
consisted of evenly spaced tones, occurring every 600 or 700 ms
(depicted in black). For the metrical sequences, the underlying meter with
a beat of 4 had a corresponding period of 600 or 700 ms, respectively.
The beat locations (unit $1,5,9$, 13) are denoted by a
induce the feeling of a metrical-beat of 4 in the listener, the strong
sequences had a phenomenally (purely due to temporal spacing)
accented tone on all four intended downbeat locations (depicted in black).
The weak sequences in contrast had an accented tone only on the first
and last downbeat (in black), but no tones on the second and third. Gray
lines denote unaccented tones, dots silent units.
[1858]

number of silent units than would be required to induce a meter of 2 or 3 , so that a meter of 4 was the most likely to be perceived based on the model of Povel and Essens (1985). The important difference between these two sets of rhythms is the strength of meter that they induce. While the strongly metrical rhythms convey a clear sense of the metrical beat, this is very hard to find in the weakly metrical rhythms. The sequences used had the same number of tones and accented beats, were composed of the same intervals, and featured the same overall meter to control for any other influences on complexity of rhythm. They corresponded to sequence numbers 706, 737, 960, and 1858 as reported by Grube and Griffiths (2009) and are depicted in Figure 1.

Across all synchronization trials, the underlying tempo of rhythms was varied between $600 \mathrm{~ms}$ and $700 \mathrm{~ms}$ - both of these have been deemed to be comfortable tapping rates (Repp, 2005) and no difference in synchronization accuracy or tapping variability was expected between these two tempi. The variation in tempo was introduced so that people would not entrain with the isochronous sequences presented first and continue tapping at this rate throughout the other trials. The starting tempo was counterbalanced between participants.

Random pitch variation was introduced throughout half of the trials to determine whether dysrhythmic participants experience further distraction given a pitch variation or not. Each sequence was presented once at a constant pitch and once with the random pitch variation. Random pitch variation was generated during the task and notes could take any semitone value within a two-octave scale. A full list of trial types is given in Table $\mathbf{1}$.
Table 1 | Characteristics of the twelve rhythms used in the synchronization tapping tasks.

\begin{tabular}{llll}
\hline Trial & Rhythm & Tempo & Random pitch \\
\hline 1 & Isochronous & 600 & No \\
2 & Isochronous & 700 & No \\
3 & Isochronous & 600 & Yes \\
4 & Isochronous & 700 & Yes \\
5 & Strong no. 706 & 600 & No \\
6 & Strong no. 737 & 700 & No \\
7 & Strong no. 706 & 600 & Yes \\
8 & Strong no. 737 & 700 & Yes \\
9 & Weak no. 960 & 600 & No \\
10 & Weak no. 1858 & 700 & No \\
11 & Weak no. 960 & 600 & Yes \\
12 & Weak no. 1858 & 700 & Yes
\end{tabular}

Rhythm numbers for strongly and weakly metrical rhythms refer to the different rhythms depicted in Figure 1. Tempo orders were counterbalanced between participants.

\section{PROCEDURE}

Spontaneous tapping data were collected for all participants before they engaged in the synchronization tasks using acoustic stimuli. Participants were asked to tap at a comfortable pace and to make 40 taps (of which the first 10 were excluded from analysis).

In the synchronization tests, the instruction was to tap out a regular beat in synchrony with the rhythmic sequences that were played. This meant that for the sequences with an isochronous beat, participants were required to tap on every auditory event. For the strongly and weakly metrical rhythms in contrast, participants were required to extract the underlying beat and tap in time with this, meaning that not every tap made would align with an auditory event. The twelve different trials as outlined in Table 1 dissociated possible effects on tapping performance of rhythm type, tempo, and variation in pitch. Participants were required to synchronize to 48 downbeats in each trial (corresponding to 12 cyclical repetitions of the sequences), with recorded responses to the first eight downbeats being discarded from analysis.

Each trial started with the presentation of eight initial beats during which the participant could listen to the stimulus and start tapping along if they wished to, but during which their tapping was not recorded. A timer bar on the screen indicated this familiarization period, at the end of which tapping started to be recorded.

\section{RESULTS}

Scores on the MBEA are given in Table 2 and demonstrate that dysrhythmic participants were scoring below threshold for rhythm subtests but above threshold for pitch subtests.

\section{SPONTANEOUS TAPPING TASK}

For the spontaneous tapping task, in which participants were asked to tap a regular beat in the absence of an acoustic stimulus, we 
Table 2 | MBEA scores for dysrhythmic participants.

\begin{tabular}{lllllll}
\hline Subject & Scale & Contour & Interval & Rhythm & $\begin{array}{l}\text { \% Correct pitch } \\
\text { (amalgamated) }\end{array}$ & \% Correct rhythm \\
\hline SS & 29 & 25 & 24 & 22 & 87 & 73 \\
SWI & 28 & 30 & 27 & 21 & 94 & 70 \\
VPO & 29 & 26 & 27 & 20 & 82 & 68 \\
\hline
\end{tabular}

Scores below 23 are considered to indicate impairments.

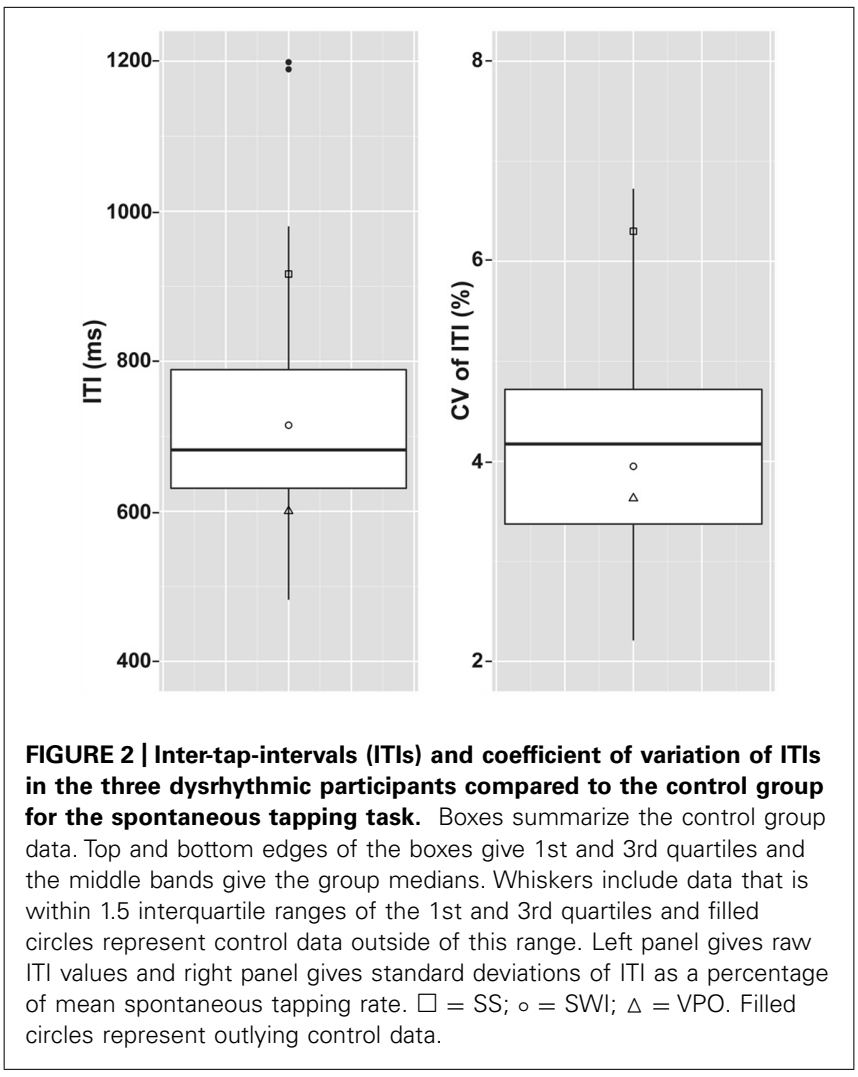

analyzed the mean tapping rate, and variability in tapping rate for each individual. The first 10 taps were excluded from analysis, in order to given an adequate "lead-in" time for participants to start tapping at a regular rate. The individual mean tapping rate (in $\mathrm{ms}$ ) was calculated for each participant. The standard deviation of the inter-tap-intervals (ITIs) for each participant was used as a measure of variance (in $\mathrm{ms}$ ), and this value was divided by the individual's mean tapping rate for that stimulus to give the coefficient of variation (CV) for spontaneous ITIs.

A summary of spontaneous tapping data from the dysrhythmic individuals compared to the controls is given in Figure 2. The dysrhythmics' data were evaluated in comparison to the control group data (after log transformation) using "singlims.exe," a program developed by Crawford and Garthwaite (2002) to evaluate single subject values relative to a control group based on Bayesian statistics. The dysrhythmics' individual mean tapping rates did not significantly differ from those of the controls; all three values were within 1.5 standard deviations of the control group's mean tapping rate. Variability in tapping rate however, was significantly different from the control group for one participant (SS; $t=1.96, p=0.029$ one tailed). Both of the other dysrhythmic participants in contrast tapped with a slightly lower average variability in comparison to the controls.

\section{SYNCHRONIZATION TASKS}

We used a circular statistics approach to derive the mean asynchrony (a measure of tap time accuracy) of synchronization trials. The onset-asynchrony for each tap was captured as an error value relative to the position of the corresponding downbeat (i.e., how late or early the participant tapped in relation to the 40 downbeat locations in the acoustic stimuli). The individual tapto-onset-asynchrony was transformed into a circular asynchrony by dividing by the most recent ITI and multiplying by 360 . Circular statistics (e.g., Mardia and Jupp, 1999) were then used to calculate the mean asynchrony for each participant in each condition and these are summarized in Figure 3. As mean asynchronies in each condition were highly variable even within control participants these were not statistically analyzed in terms of differences between dysrhythmics and controls.

To assess the ability to tap at the intended rate along to the stimuli we calculated the mean tapping rate and regularity of tapping rate. This was done by first dividing ITIs by the tempo of the trial to give relative tapping rates and enable comparison across the two tempi (excluding all outliers of more than 2.5 standard deviations from the sample mean ITI). One control participant's data was excluded entirely, due to two trials in which all ITIs were outliers relative to the remaining participants' data. Mean relative tapping rates for each condition are summarized in Figure 4. The data demonstrate that control participants tapped very close to the expected rate in most conditions, although displaying a large amount of variability in the weakly metrical conditions.

The standard deviation of relative tapping rate (as calculated above) was used to measure an individual's tapping stability within a trial. After log transformation in order to obtain normally distributed data samples, control data were compared across different conditions using a 3 (rhythm type: isochronous, strong, weak) $\times 2$ (pitch: random variation, no pitch change) ANOVA, with Greenhouse-Geisser correction for non-sphericity. A significant main effect of rhythm condition was identified, $F(2,70)=21, p<0.0001, \mathrm{G}^{2}=0.17$, no main effect of pitch $(p=0.41)$, and a near significant interaction between the two, $F(2,70)=2.97, p=0.058, \mathrm{G}^{2}=0.012$. Post hoc pairwise $t$-tests comparing the three rhythm types, with Bonferroni 
Isochrony, no pitch change

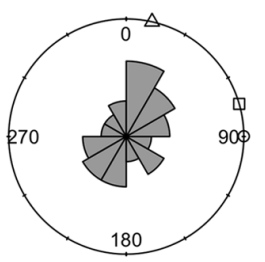

Strong, no pitch change

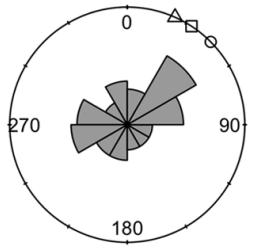

Weak, no pitch change

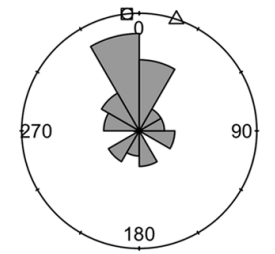

Isochrony, pitch change

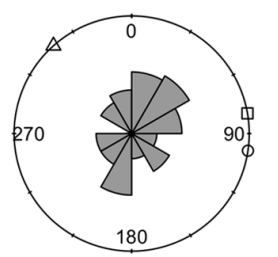

Strong, pitch change

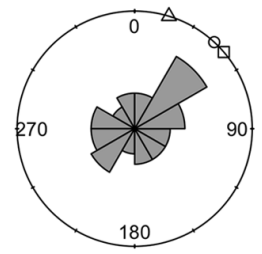

Weak, pitch change

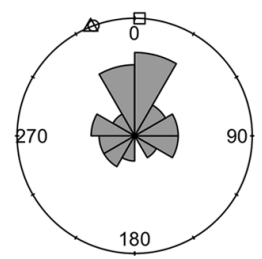

FIGURE 3 | Mean circular asynchronies for the three dysrhythmic participants compared to the control group in all six conditions of the synchronization task. Circular histograms depict frequency density of mean asynchronies around a circle. Values close to $0^{\circ}$ indicate tapping close to the correct tap time, while values close to $180^{\circ}$ indicate tapping halfway between the correct times. $\square=\mathrm{SS} ; \circ=\mathrm{SWl} ; \Delta=\mathrm{VPO}$.

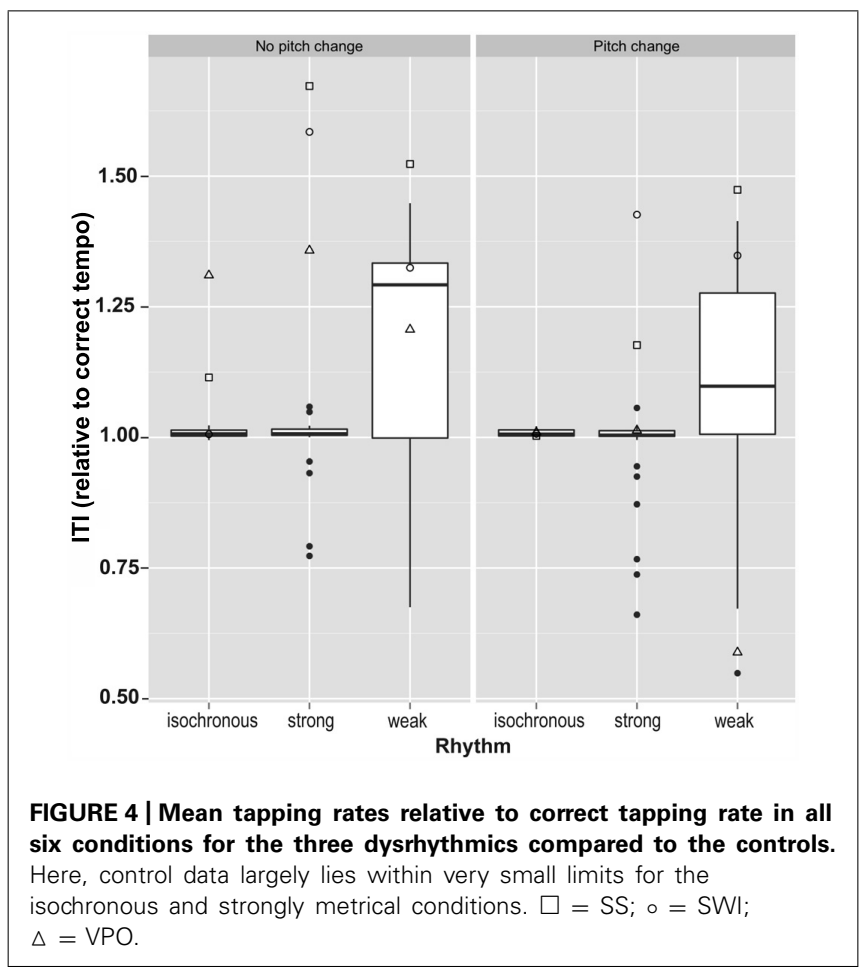

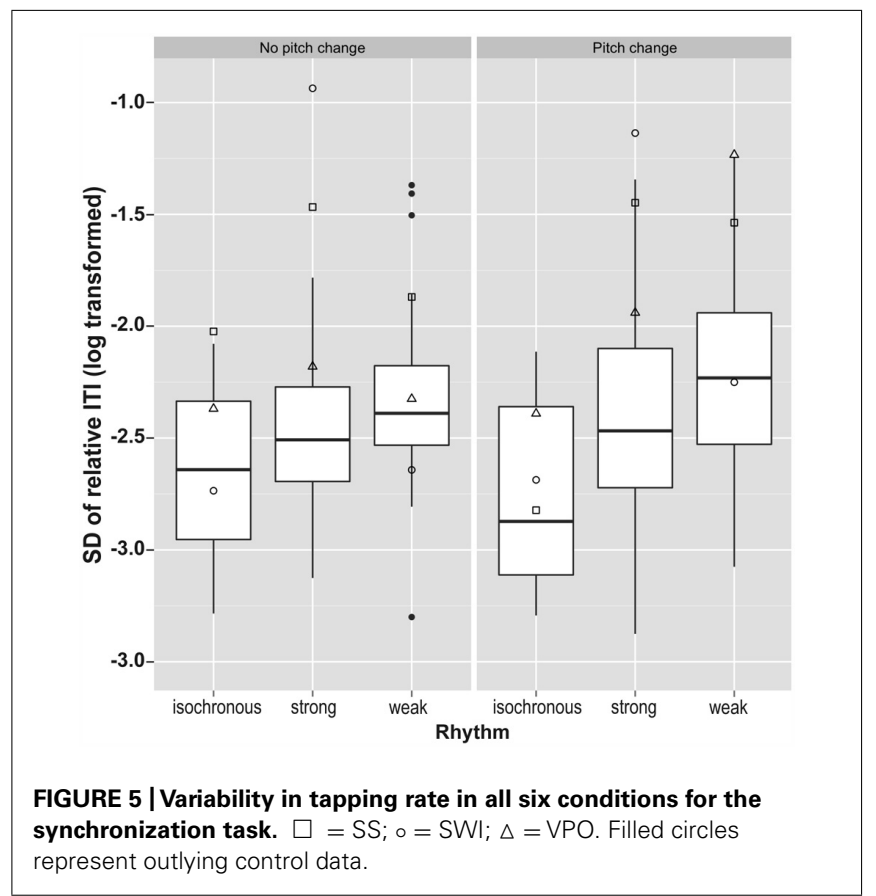

correction for multiple comparison, demonstrated that variability in tapping rate differed significantly between all three rhythm types. Performance for isochronous rhythms exhibited significantly smaller variability in tapping rate than for both strongly metrical rhythms $(p<0.0001)$ and weakly metrical rhythms $(p<0.0001)$, while strongly metrical rhythms demonstrated significantly lower variability in tapping rate compared to weakly metrical rhythms $(p=0.001)$. These results are summarized in Figure 5.

The dysrhythmics' synchronization tapping data exhibited a qualitative difference in performance, identifiable at a gross level; while all three dysrhythmics often produced the correct number of taps for the isochronous sequences, they typically produced only half as many taps as there were downbeats for the strongly metrical rhythms. This might indicate that the dysrhythmics were tapping at half the tempo of the intended meter. The number of "missed beats," i.e., the discrepancy between the 40 presented downbeats in the stimuli and the number of taps produced on each trial, are given in Table 3, along with mean ITIs for dysrhythmics compared to controls. The numbers support the notion that dysrhythmics tended to tap at half the expected tempo, which did not occur in the control data. In addition, as can be seen in Figure 5, variability in tapping rate for dysrhythmics was higher than in controls in these conditions, which may be a consequence of tapping at a much slower tempo than intended. Owing to this assumed difference in hierarchical level with which the dysrhythmics compared to the control participants synchronized their tapping, we refrained from performing formal statistical analysis on the synchronization accuracy and variability measures. Figure 6 gives raw tapping data for dysrhythmics compared to one randomly selected control subject in Trials 5 and 7, to demonstrate the dysrhythmics tendency to tap regularly, but at a slower tempo corresponding to the next-higher hierarchical level than intended. 
Table 3 | Number of missed beats and mean ITls in the dysrhythmic participants compared to the control group.

\begin{tabular}{|c|c|c|c|c|c|c|c|c|c|c|c|c|c|}
\hline Measure & Participant & Trial 1 & Trial 2 & Trial 3 & Trial 4 & Trial 5 & Trial 6 & Trial 7 & Trial 8 & Trial 9 & Trial 10 & Trial 11 & Trial 12 \\
\hline Missed & SS & 0 & 0 & 9 & 0 & 23 & 11 & 19 & 19 & * & 17 & 13 & 17 \\
\hline \multirow{2}{*}{ taps } & VPO & 1 & 0 & 0 & 7 & 0 & 1 & 19 & 0 & 11 & 12 & 6 & 12 \\
\hline & Control mean & 0.05 & 0.29 & 0.05 & 0.11 & 0.87 & 1.16 & 0.87 & 0.26 & 6.32 & 5.97 & 6.76 & 6.47 \\
\hline Mean & SS & 600 & 704 & 770 & 701 & 1294 & 389 & 1178 & 1366 & $*$ & 1150 & 882 & 1164 \\
\hline \multirow{2}{*}{ ITI (ms) } & VPO & 614 & 713 & 600 & 1223 & 601 & 717 & 1140 & 721 & 337 & 381 & 723 & 386 \\
\hline & Control mean & 604 & 713 & 606 & 707 & 595 & 678 & 596 & 700 & 718 & 816 & 703 & 818 \\
\hline
\end{tabular}

Numbers in bold indicate that subjects miss out approximately half of the expected beats. *indicates missing data.

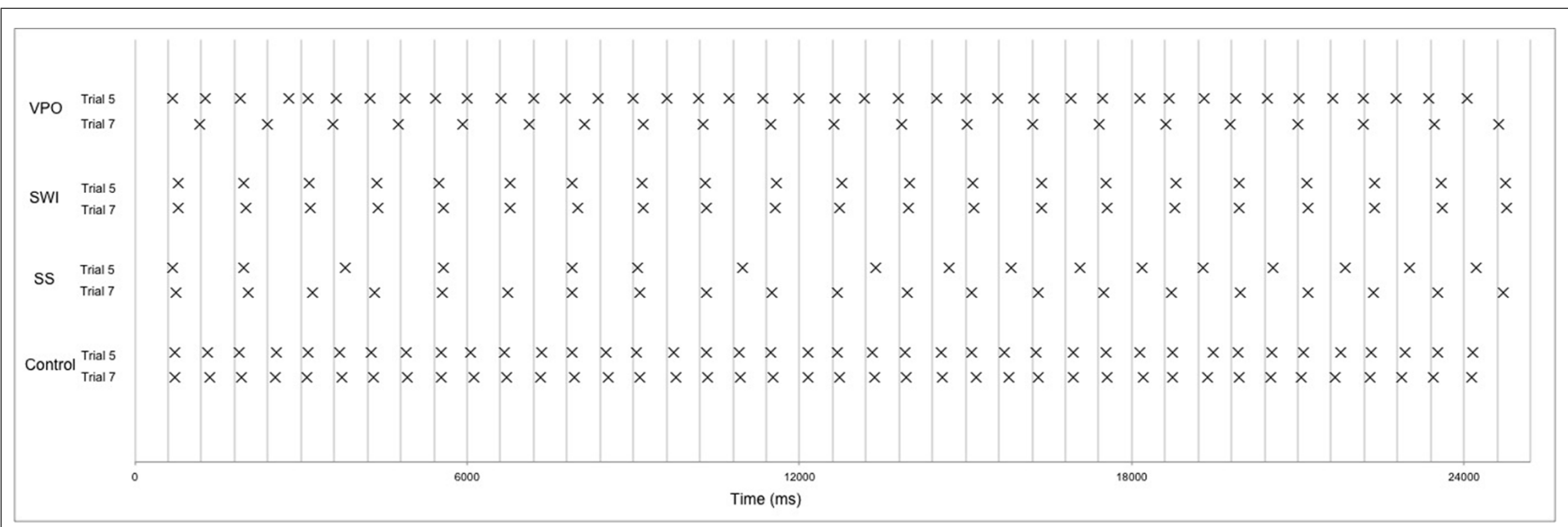

FIGURE 6 | Raw tapping data in Trial 5 and Trial 7 for dysrhythmics and one control subject (randomly selected). Lines give the correct tap times, and the midpoint of each $\times$ represents an individual tap. Taps corresponding to the first 40 tones are given here.

In weakly metrical trials, performance was comparably poor for all participants, with beats being "missed" frequently in both participant groups. It is clear from Figure 4 furthermore, that control participants were not always tapping with the expected tempo. This problem in tapping at the expected tempo is likely to reflect the fact that even controls have problems extracting the meter in these trials thus the poor performance of dysrhythmics in these conditions is unsurprising.

\section{DISCUSSION}

The current study investigates individuals with what we are terming "dysrhythmia": a congenital, selective deficit in rhythm perception and production. This deficit appears to be much rarer than the form of amusia that has been characterized as a selective pitch impairment. Importantly, the dysrhythmic participants studied here did not demonstrate a general problem with spontaneous tapping, indicating that these individuals are unlikely to suffer from motor deficits that could explain synchronization performance. Their difficulties with tapping along to the beat of different types of rhythm are thus likely to specifically relate to issues with extracting rhythmic information. In the present cases, both rhythm perception and production tasks revealed anomalies relative to the performance of the controls, including both an impairment in musical rhythm perception, measured via the MBEA, and abnormal tapping behavior when required to extract the beat from a rhythmic sequence.

If beat-based rhythm production was generally impaired in dysrhythmic subjects then we would expect to also find difficulties in the maintenance of a self-paced steady beat. However, no difference in spontaneous tapping rate was found between control subjects and the dysrhythmics, apart from slightly larger variability in tapping rate for one dysrhythmic compared to the controls. Overall, the normal ability of these individuals to produce a self-paced steady beat demonstrates that rhythmic difficulties cannot be ascribed to motor deficits with generating and maintaining a steady beat. One might expect, therefore, that dysrhythmics would be able to perform normally if required to continue tapping out an isochronous beat after entraining with an acoustic stimulus. Their problem seems to lie specifically in extracting the correct (intended) meter from nonisochronous metrical rhythms. This differentiation merits future investigation. 
Consistent with the notion of a close-to normal ability to produce a steady beat, basic sensorimotor entrainment was largely preserved: for synchronization with an isochronous beat, tapping rates in dysrhythmic participants (Table 3 ) demonstrate that they were mostly tapping at the intended tempo, as expected in normal participants and seen in the controls. When required to extract a metrical-beat and synchronize to it, in contrast, the dysrhythmics' tapping behavior deviated significantly from that of the controls. For the strongly metrical sequences, the dysrhythmics produced approximately half of the beats required for the intended downbeat, suggesting that they had difficulties extracting a regular beat from a rhythm (something controls can do with ease). In weakly metrical conditions all participants performed badly, suggesting a floor effect for these rhythm sequences.

The finding of entraining at an unexpected metrical level is very similar to that of Dalla Bella and Peretz (2003) who studied congenitally amusic subjects with pitch-based deficits, and this similarity suggests that there may be some patterns of impairment that generalize between these groups. Entraining at a higher metrical level can occur because subjective grouping of tones in metrical sequences is possible in a number of ways depending on which level of the metrical-beat hierarchy one perceives as the most prominent and comfortable one. For a strongly metrical rhythm such as in the sequences used here, up to three potential levels of perception of the beat exist: one at half, one at twice, and one at the tempo of the intended dominant meter. According to behavioral literature of the past decades promoting a range from a minimum of 200 to up to about 1000 ms (e.g., see Repp, 2005), people would generally entrain at the intended meter of 600 or 700 ms. However, humans tend to be quite capable of tapping along in time to music at a range of tempos, and the criteria used to evaluate this ability in musically oriented work has typically been based on whether successive taps coincide with beats in the music, regardless of the hierarchical level this occurs at (Drake and Bertrand, 2001). Whether the current finding is due to a rate-based limitation (i.e., slowing-down of the processing of metrical periodicity) or a deficit in multilevel hierarchical processing of metrical structure remains to be explored.

Either way, this problem is quite different from "poor synchronizers" as identified by Sowinski and Dalla Bella (2013), who exhibit poor synchronization as a consequence of error correction difficulties. Participants in their study did not have problems with rhythm perception, although some may have experienced synchronization problems as a consequence of poor pitch perception. The heterogeneity of synchronization disorders point to the complexity of the processes underlying this ability, and suggests that much further work is required to find out how these problems arise.

To our knowledge, this is the first report of a type of musical deficit that demonstrates impaired rhythm perception and beat extraction in the face of intact pitch perception, in neurologically intact individuals. This pattern is the opposite of that which has previously been reported in individuals termed congenitally amusic, according to the MBEA, where as many as half are reported to have pitch deficits in the face of normal scores on the rhythm test (Ayotte et al., 2002). We suggest that the findings from these individuals contribute to an emerging picture of the different ways in which rhythm perception can be compromised, with theoretical implications for our understanding of the processing of rhythm in the typical population.

\section{REFERENCES}

Ayotte, J., Peretz, I., and Hyde, K. (2002). Congenital amusia a group study of adults afflicted with a music-specific disorder. Brain 125, 238-251. doi: 10.1093/brain/awf028

Beebe, B., and Lachmann, F. M. (1988). The contribution of mother-infant mutual influence to the origins of self-and object representations. Psychoanal. Psychol. 5, 305-337. doi: 10.1037/0736-9735.5.4.305

Bernieri, F. J., Reznick, J. S., and Rosenthal, R. (1988). Synchrony, pseudosynchrony, and dissynchrony: measuring the entrainment process in mother-infant interactions. J. Pers. Soc. Psychol. 54, 243-253. doi: 10.1037/0022-3514.54 2.243

Crawford, J. R., and Garthwaite, P. H. (2002). Investigation of the single case in neuropsychology: confidence limits on the abnormality of test scores and test score differences. Neuropsychologia 40, 1196-1208. doi: 10.1016/S00283932(01)00224-X

Dalla Bella, S., and Peretz, I. (2003). Congenital amusia interferes with the ability to synchronize with music. Ann. N. Y. Acad. Sci. 999, 166-169. doi: 10.1196/annals.1284.021

Dissanayake, E. (2000). "Antecedents of the temporal arts in early mother-infant interaction," in The Origins of Music, eds N. L. Wallin, B. R. Merker, and S. Brown (Cambridge, Mass.: MIT Press), 389-410.

Drake, C., and Bertrand, D. (2001). The quest for universals in temporal processing in music. Ann. N. Y. Acad. Sci. 930, 17-27. doi: 10.1111/j.17496632.2001.tb05722.x

Ehrle, N., and Samson, S. (2005). Auditory discrimination of anisochrony: influence of the tempo and musical backgrounds of listeners. Brain Cogn. 58, 133-147. doi: 10.1016/j.bandc.2004.09.014

Farrugia, N., Benoit, C. E., Harding, E., Kotz, S. A., and Dalla Bella, S. (2012). BAASTA: battery for the assessment of auditory sensorimotor and timing abilities. Inst. Hum. Cogn. Brain Sci. 18, 9.

Foxton, J. M., Nandy, R. K., and Griffiths, T. D. (2006). Rhythm deficits in "Tone deafness". Brain Cogn. 62, 24-29. doi: 10.1016/j.bandc.2006.03.005

Fraisse, P. (1963). The Psychology of Time. Oxford, UK: Harper \& Row.

Friberg, A., and Sundberg, J. (1995). Time discrimination in a monotonic, isochronous sequence. J. Acoust. Soc. Am. 98, 2524-2531. doi: 10.1121/1.413218

Grube, M., and Griffiths, T. D. (2009). Metricality-enhanced temporal encoding and the subjective perception of rhythmic sequences. Cortex 45, 72-79. doi: 10.1016/j.cortex.2008.01.006

Grube, M., Kumar, S., Cooper, F. E., Turton, S., and Griffiths, T. D. (2012). Auditory sequence analysis and phonological skill. Proc. R. Soc. B Biol. Sci. 279, 4496-4504. doi: $10.1098 / \mathrm{rspb} .2012 .1817$

Handel, S. (1998). The interplay between metric and figural rhythmic organization. J. Exp. Psychol. Hum. Percept. Perform. 24, 1546-1561. doi: 10.1037/00961523.24.5.1546

Hannon, E. E., and Trehub, S. E. (2005). Tuning in to musical rhythms: infants learn more readily than adults. Proc. Natl. Acad. Sci. U.S.A. 102, 12639-12643. doi: 10.1073/pnas.0504254102

Hebert, S., and Cuddy, L. L. (2002). Detection of metric structure in auditory figural patterns. Percept. Psychophys. 64, 909-918. doi: 10.3758/BF03196795

Hirsh, I. J., Monahan, C. B., Grant, K. W., and Singh, P. G. (1990). Studies in auditory timing: 1. Simple patterns. Percept. Psychophys. 47, 215-226. doi: 10.3758/BF03204997

Hove, M. J., and Risen, J. L. (2009). It's all in the timing: interpersonal synchrony increases affiliation. Soc. Cogn. 27, 949-960. doi: 10.1521/soco.2009.27.6.949

Huss, M., Verney, J. P., Fosker, T., Mead, N., and Goswami, U. (2011) Music, rhythm, rise time perception and developmental dyslexia: perception of musical meter predicts reading and phonology. Cortex 47, 674-689. doi: 10.1016/j.cortex.2010.07.010

Hyde, K. L., and Peretz, I. (2004). Brains that are out of tune but in time. Psychol. Sci. 15, 356-360. doi: 10.1111/j.0956-7976.2004.00683.x

Iversen, J. R., and Patel, A. D. (2008). "The Beat Alignment Test (BAT): surveying beat processing abilities in the general population," in Proceedings of the 
$10^{\text {th }}$ International Conference on Music Perception and Cognition (ICMPC10), (Sapporo: Casual Productions), 465-468.

Jusczyk, P. W., Hirsh-Pasek, K., Nelson, D. G., Kennedy, L. J., Woodward, A., and Piwoz, J. (1992). Perception of acoustic correlates of major phrasal units by young infants. Cogn. Psychol. 24, 252-293. doi: 10.1016/0010-0285(92)90009-Q

Jusczyk, P. W., Houston, D. M., and Newsome, M. (1999). The beginnings of word segmentation in English-learning infants. Cogn. Psychol. 39, 159-207. doi: 10.1006/cogp.1999.0716

Knoblich, G., Butterfill, S., and Sebanz, N. (2011). Psychological research on join action: theory and data. Psychol. Learn. Motiv. 54, 59-101. doi: 10.1016/B978-0 12-385527-5.00003-6

Konvalinka, I., Xygalatas, D., Bulbulia, J., Schjødt, U., Jegindø, E. M., Wallot S., et al. (2012). Synchronized arousal between performers and related spectators in a fire-walking ritual. Proc. Natl. Acad. Sci. 108, 8514-8519. doi: 10.1073/pnas.1016955108

Launay, J., Dean, R. T., and Bailes, F. (2013). Synchronization can influence trust following virtual interaction. Exp. Psychol. 60, 53-63. doi: 10.1027/1618$3169 / \mathrm{a} 000173$

London, J. (2004). Hearing in Time. New York: Oxford University Press. doi: 10.1093/acprof:oso/9780195160819.001.0001

Mardia, K. V., and Jupp, P. E. (1999). Directional Statistics. Chichester: Wiley doi: $10.1002 / 9780470316979$

Monahan, C. B., and Hirsh, I. J. (1990). Studies in auditory timing: 2. Rhythm patterns. Percept. Psychophys. 47, 227-242. doi: 10.3758/BF03204998

Oullier, O., De Guzman, G. C., Jantzen, K. J., Lagarde, J., and Kelso, J. A. S. (2008). Social coordination dynamics: measuring human bonding. Soc. Neurosci. 3, 178 192. doi: $10.1080 / 17470910701563392$

Patel, A. D., Iversen, J. R., Chen, Y., and Repp, B. H. (2005). The influence of metricality and modality on synchronization with a beat. Exp. Brain Res. 163 226-238. doi: 10.1007/s00221-004-2159-8

Peretz, I., Champod, A. S., and Hyde, K. (2003). Varieties of musical disorders. Ann. N. Y. Acad. Sci. 999, 58-75. doi: 10.1196/annals. 1284.006

Peretz, I., and Hyde, K. L. (2003). What is specific to music processing? Insights from congenital amusia. Trends Cogn. Sci. 7, 362-367. doi: 10.1016/S13646613(03)00150-5

Phillips-Silver, J., Toiviainen, P., Gosselin, N., PichẼ, O., Nozaradan, S., Palmer C., et al. (2011). Born to dance but beat deaf: a new form of congenital amusia. Neuropsychologia 49, 961-969. doi: 10.1016/j.neuropsychologia.2011.02.002

Phillips-Silver, J., and Trainor, L. J. (2005). Feeling the beat: movement influences infant rhythm perception. Science 308, 1430-1430. doi: 10.1126/science.1110922

Povel, D. J., and Essens, P. (1985). Perception of temporal patterns. Music Percept. 2, 411-440. doi: 10.2307/40285311

Praamstra, P., Turgeon, M., Hesse, C., Wing, A., and Perryer, L. (2003). Neurophysiological correlates of error correction in sensorimotor-synchronization. Neuroimage 20, 1283-1297. doi: 10.1016/S1053-8119(03)00351-3
Repp, B. H. (2005). Sensorimotor synchronization: a review of the tapping literature. Psychon. Bull. Rev. 12, 969-992. doi: 10.3758/BF03206433

Smith, M. R., Cutler, A., Butterfield, S., and Nimmo-Smith, I. (1989). The perception of rhythm and word boundaries in noise-masked speech. J. Speech Hear. Res. 32 912-920.

Sowinski, J., and Dalla Bella, S. (2013). Poor synchronization to the beat may result from deficient auditory-motor mapping. Neuropsychologia 51, 1952-1963. doi: 10.1016/j.neuropsychologia.2013.06.027

ten Hoopen, G., Boelaarts, L., Gruisen, A., Apon, I., Donders, K., Mul, N., etal. (1994). The detection of anisochrony in monaural and interaural sound sequences. Percept. Psychophys. 56, 110-120. doi: 10.3758/BF0321 1694

Thaut, M. H., and Abiru, M. (2010). Rhythmic auditory stimulation in rehabilitation of movement disorders: a review of the current research. Music Percept. Interdiscip. J. 27, 263-269. doi: 10.1525/mp.2010.27. 4.263

Vickhoff, B., Malmgren, H., Åström, R., Nyberg, G., Ekström, S.-R., Engwall, M., et al. (2013). Music structure determines heart rate variability of singers. Front Psychol. 4:334. doi: 10.3389/fpsyg.2013.00334

Wiltermuth, S. S., and Heath, C. (2009). Synchrony and cooperation. Psychol. Sci. 20, 1-5. doi: 10.1111/j.1467-9280.2008.02253.x

Wing, A., and Kristofferson, A. (1973). Response delays and the timing of discrete motor responses. Percept. Psychophys. 14, 5-12. doi: 10.3758/BF031 98607

Zentner, M., and Eerola, T. (2010). Rhythmic engagement with music in infancy. Proc. Natl. Acad. Sci. 107, 5768-5773. doi: 10.1073/pnas.1000 121107

Conflict of Interest Statement: The authors declare that the research was conducted in the absence of any commercial or financial relationships that could be construed as a potential conflict of interest.

Received: 15 July 2013; paper pending published: 08 November 2013; accepted: 08 January 2014; published online: 05 February 2014.

Citation: Launay J, Grube M and Stewart L (2014) Dysrhythmia: a specific congenital rhythm perception deficit. Front. Psychol. 5:18. doi: 10.3389/fpsyg.2014.00018

This article was submitted to Auditory Cognitive Neuroscience, a section of the journal Frontiers in Psychology.

Copyright (c) 2014 Launay, Grube and Stewart. This is an open-access article distributed under the terms of the Creative Commons Attribution License (CC BY). The use, distribution or reproduction in other forums is permitted, provided the original author(s) or licensor are credited and that the original publication in this journal is cited, in accordance with accepted academic practice. No use, distribution or reproduction is permitted which does not comply with these terms. 